\title{
Zur Realisierbarkeit europäischer Visionen - Direkte Demokratie als Förderprogramm europäischer Demokratie
}

Harald Eberhard/Konrad Lachmayer

\section{Direkte Demokratie im europäischen Demokratisierungsprozess - eine Annäherung}

Setzt man sich mit direkter Demokratie auf europäischer Ebene auseinander, stellt sich zuallererst die Frage nach Demokratie an sich. Nationalstaatliche Kategorien sind dabei nur begrenzt verwertbar, stellen aber einen wesentlichen Bestandteil des sich historisch entwickelnden Demokratieverständnisses dar. Der erste Schritt der Analyse direkter Demokratie besteht daher in der Auseinandersetzung mit dem Demokratieprozess auf europäischer Ebene (2.). In einem zweiten und zentralen Schritt ist die Rolle direkter Demokratie in diesem europäischen Demokratieprozess zu verorten (3.). In einem dritten und abschließenden Schritt sind neue Perspektiven direkter Demokratie als post-nationales Phänomen anzudenken. (4.)

\section{Europäische Demokratie - welche Demokratie?}

Versteht man direkte Demokratie als Alternative zur oder Ergänzung von repräsentativer Demokratie, so erhebt sich zunächst die Frage, welches Demokratiemodell die Europäische Union (EU) selbst verwirklicht und inwieweit man die Charakteristika des Zusammenspiels repräsentativer und direkter Demokratie auf europäischer Ebene rekonstruieren kann. Die auf nationaler Ebene in den unterschiedlichen Intensitäten und Formen gefundenen Modelle entwickeln sich auf europäischer Ebene jedenfalls eigenständig. ${ }^{1}$

\section{a) Die „Konstitutionalisierung“ Europas}

Mit der Frage nach der europäischen Demokratie steht nicht so sehr die Frage der „Staatsqualität“ der EU im Mittelpunkt. Demokratie, sieht man sie als Postulat einer bestimmten Ausgestaltung und Legitimation von Rechtsetzung, steht in keinem zwingenden Zusammenhang mit Staatlichkeit. ${ }^{2}$ Es steht fest, dass die in den letzten

1 Treffend dazu H. Blanke, Essentialia einer Europäischen Verfassungsurkunde, ThürVB1. 2002, 197 (227): „Die Anforderungen an die künftige demokratische Kultur der Union sind entsprechend der Einzigartigkeit dieses Gebildes in eigenständiger Weise, also unter Vermeidung einer ,blinden Übertragung innerstaatlicher Denkmuster', auszugestalten.“

2 Vgl. dazu G. Lübbe-Wolff, Volk, Demokratie, Verfassung - Die „Verfassung für Europa“ als Herausforderung an die Verfassungstheorie, in: W. Kluth (Hrsg.), Europäische Integration und nationales Verfassungsrecht, 2007, S. 47 (58 ff.). Siehe dazu auch U. Volkmann, Setzt Demo- 
Jahrzehnten erfolgten Änderungen des positiven Unions- und Gemeinschaftsrechts, insb. der Gemeinschaftsverträge, einen Prozess darstellen, der auch von einer entsprechenden Entwicklung der Demokratietheorie begleitet wurde und wird, für die die Europäisierung aufgrund ihrer Geschwindigkeit, aber auch aufgrund der nach wie vor offenen Finalität ${ }^{3}$ Europas bedeutsame Herausforderungen mit sich gebracht hat. ${ }^{4}$ Die besondere Facette dieses demokratischen Aspekts liegt in der Legitimation des hoheitlichen Handelns der EU, das für jeden Einzelnen verbindliche Normen zu kreieren imstande ist. ${ }^{5}$ Die EU als supranationaler Hoheitsträger ist nicht nur ,demokratiefähig“, sie ist auch „demokratiebedürftig“, wie die zuweilen getroffene Feststellung eines „Demokratiedefizits“6 eingehend belegt. Dies gilt im Übrigen auch für das Element der Rechtsstaatlichkeit. ${ }^{7}$ Die EU stellt - völlig unabhängig von

kratie den Staat voraus?, AöR 128 (2002), 575 (583 ff., insb. 585): „Der Satz, die Europäische Union sei mangels europäischen Volkes nicht demokratiefähig, beruht ... auf einer Vermengung der verschiedenen Bedeutungsschichten des Volksbegriffs und mag zwar auf bestimmte tatsächliche Voraussetzungen für eine funktionierende Demokratie verweisen, nicht aber auf einen begrifflichen Zusammenhang mit dem Staat.“

$3 \mathrm{Zu}$ den verschiedenen Finalitätskonzepten vgl. etwa U. Haltern, Gestalt und Finalität, in: A. v. Bogdandy/J. Bast (Hrsg.), Europäisches Verfassungsrecht, 2. Aufl. 2009, S. 279 (285 ff.); I. Pernice, Zur Finalität Europas, in: G.F. Schuppert/I. Pernice/U. Haltern (Hrsg.), Europawissenschaft, 2005, S. 743 (746).

4 Eingehend zu diesen Herausforderungen etwa M. Nettesheim, Demokratisierung der Europäischen Union und Europäisierung der Demokratietheorie - Wechselwirkungen bei der Herausbildung eines europäischen Demokratieprinzips, in: H. Bauer/P.M. Huber/K.-P. Sommermann (Hrsg.), Demokratie in Europa, 2005, S. 143 (165 f.): „Die Entwicklung der Theorie supranationaler Demokratie ... muß sich in einem dialektischen Prozeß abspielen, in dem überkommene Vorstellungen über die demokratische Legitimation von Herrschaft, Einsichten in die tatsächlichen Besonderheiten der EU und Überlegungen zu den Funktionsnotwendigkeiten überstaatlicher Vergemeinschaftung in einen - fruchtbaren - Austauschprozeß treten. So wenig zu erwarten ist, daß sich in diesem Prozeß ein unionales Demokratieprinzip herausbilden wird, das jeden Bezug und jede Ähnlichkeit zu den nationalstaatlichen Parallelprinzipien abgeworfen hat, so wenig ist zu erwarten, daß sich die Demokratie in der EU einmal eins zu eins mit nationalstaatlicher Demokratie gleichsetzen lassen wird. ... Vor diesem Hintergrund muß sich die Demokratietheorie der Frage annehmen, wo sie sich öffnen und anpassen muß, um den Prozeß der Integration ... einfangen und normativ begleiten zu können. Sie muß sich vor allem damit auseinandersetzen, wo sich in der Ordnung des Integrationsverbands Ansätze finden, mit denen der normative Anspruch politisch-demokratischer Selbstbestimmung besser eingelöst werden kann als im nationalstaatlichen Kontext. Dabei hat die Demokratietheorie nicht nur mit der Schwierigkeit zu kämpfen, daß die Struktur der EU durchaus amorph ist; vielleicht noch größer ist die Schwierigkeit, die sich daraus ergibt, daß sich die EU in schneller Entwicklung befindet." Siehe dazu auch M. Nettesheim, Developing a Theory of Democracy for the European Union, Berkeley Journal of International Law 23 (2005), $358 \mathrm{ff}$.

5 Dazu etwa H. Klein, Europäisches Parteienrecht, in: FS Ress, 2005, S. 541 (543).

6 Dazu etwa T. Öhlinger, EU-BeitrittsBVG, in: K. Korinek/M. Holoubek (Hrsg.), Österreichisches Bundesverfassungsrecht Kommentar, 1. Lfg. 1999, Rz. 84 f. m.w.N.

7 Dazu näher H. Eberhard, Das Legalitätsprinzip im Spannungsfeld von Gemeinschaftsrecht und nationalem Recht. Stand und Perspektiven eines ,europäischen Legalitätsprinzips“, ZÖR 63 (2008), 49 (53 ff.). 
staatlichen Kategorien - ein demokratie- und rechtsstaatsfähiges sowie -bedürftiges Gebilde dar. ${ }^{8}$

Demgegenüber in einem entscheidenden Zusammenhang stehen die Begriffe der „Konstitutionalisierung“ Europas und der europäischen Demokratie. Die sich vor allem auf die Ebene des Primärrechts beziehende Konstitutionalisierung Europas operiert mit einem Verfassungsbegriff, ${ }^{9}$ der die Unterschiede zum nationalen ${ }^{10}$ Verfassungsbegriff nicht negiert, aber jene Elemente herausfiltert, deren Realisierung überall dort notwendig ist, wo es um die Regelung der Rahmenbedingungen von Rechtsetzung und damit von politischer Machtausübung geht. Die Schaffung einer

8 Eberhard, Legalitätsprinzip (Fn. 7), S. 55. Siehe dazu - wenn auch mit Blick auf die „Defizite“ - etwa Nettesheim, Demokratisierung (Fn. 4), S. 165 ff.; S. Kadelbach, Bedingungen einer demokratischen Europäischen Union, EuGRZ 2006, 384 (385 ff.); R. Wahl, Erklären staatstheoretische Leitbegriffe die Europäische Union?, JZ 2005, 916 (922 ff.), der speziell für die Rechtsstaatlichkeit festhält (922): „Rechtsstaatlichkeit läßt sich ... unschwer generalisieren und unabhängig von der konkreten Gestalt einer politischen Einheit verstehen." Ebenso C. Calliess, in: C. Calliess/M. Ruffert (Hrsg.), EUV/EGV Kommentar, 3. Aufl. 2007, Art. 6 EUV Rn. 20: „Das Rechtsstaatsprinzip entstammt zwar den Rechtsordnungen der Mitgliedstaaten, ist aber auf die Union übertragbar, auch wenn diese selbst keine Staatsqualität besitzt“". Im Hinblick auf die Demokratie weist $R$. Wahl (ebda., S. 924) auf den Umstand hin, dass man eine „Gesamtkonstellation von (reduzierter) Demokratie in den nationalen Staaten plus Demokratie in der EU“ in den Blick zu nehmen hat. Zur Entkoppelung von Demokratie und Rechtsstaatlichkeit vom Staatsbegriff siehe auch J. Busch, Existiert Europa? - Antworten der Verfassungstheorie, in: H. Eberhard/K. Lachmayer/G. Thallinger (Hrsg.), Reflexionen zum Internationalen Verfassungsrecht, 2005, S. 87 (98 ff.).

9 Dass die EU bislang keine Verfassung im (formellen) „Vollsinn“ dieses Begriffes besitzt, steht außer Frage: vgl. dazu etwa T. Öhlinger, Braucht Europa eine Verfassung?, JRP 2002, 37 (39 f.). - Zur Ähnlichkeit der Verfassungsbegriffe in inhaltlicher (materiell-rechtlicher) Hinsicht und zur Verfassungseigenschaft des Primärrechts in einem materiellen Sinn siehe jedoch T. Öhlinger, Der souveräne Nationalstaat als Akteur im Prozess der Konstitutionalisierung aus verfassungsrechtlicher Sicht, in: E. Busek/W. Hummer (Hrsg.), Die Konstitutionalisierung der Verbandsgewalt in der (neuen) Europäischen Union, 2006, S. 109 (110 f.); P. Pernthaler, Österreichisches Bundesstaatsrecht, 2004, S. 34; R. Streinz/C. Ohler/C. Herrmann, Die neue Verfassung für Europa, 2005, S. 5 f.; H. Hofmann, Zu Entstehung, Entwicklung und Krise des Verfassungsbegriffs, in: FS Häberle, 2004, S. 157 (169). Zur Rolle des Primärrechts als Verfassung vgl. schon EuGH, Les Verts, Slg. 1986, 1339, Rz. 23 („Vertrag als Verfassungsurkunde der Gemeinschaft" ). - Zur notwendigen Abkoppelung des Verfassungsbegriffes von der Staatlichkeit S. Griller, Die Europäische Union - Ein staatsrechtliches Monstrum?, in: G.F. Schuppert/I. Pernice/U. Haltern (Hrsg.), Europawissenschaft, 2005, S. 201 (235 ff.). Zur Divergenz zwischen der nicht vorhandenen Verfassunggebung im formalen, verfahrensmäßigen Sinne und der sich - gerade im Hinblick auf rechtsstaatliche Kategorien - intensivierenden und expandierenden Verfassung im materiellen Sinne auf europäischer Ebene siehe P. Häberle, Europäische Verfassungslehre, 4. Aufl. 2006, S. 633. Zum notwendigen weiten Verfassungsbegriff des Internationalen Verfassungsrechts vgl. H. Eberhard/K. Lachmayer/G. Thallinger, Über Inhalt und Methode des Internationalen Verfassungsrechts als Wissenschaftsdisziplin, in: dies., Reflexionen (Fn. 8), S. 175 (180 ff.).

$10 \mathrm{Zu}$ den unterschiedlichen nationalstaatlichen Verfassungsbegriffen vgl. etwa Pernthaler, Bundesstaatsrecht (Fn. 9), S. 29 ff.; B. Wieser, Vergleichendes Verfassungsrecht, 2005, S. 49 f. Zur grundlegenden Unterscheidung zwischen dem formellen und materiellen Verfassungsbegriff im nationalstaatlichen Kontext siehe F. Koja, Allgemeine Staatslehre, 1993, S. 105 ff. 
europäischen Verfassung ist nicht als „Fixum“, sondern als „Prozess“ erkannt worden. ${ }^{11}$ Vornehmlicher Anknüpfungspunkt dieses Prozesses waren die sukzessive erfolgten Erweiterungen der Kompetenzen des Europäischen Parlaments (EP). ${ }^{12}$ Der Demokratisierungsprozess der EU erweist sich aus diesem Grund auch als „Parlamentarisierung". ${ }^{13}$

Der prozesshafte Charakter der Demokratisierung impliziert - versteht man Demokratie mit $N$. Petersen als teleologisches Prinzip - zwei wichtige Konsequenzen. ${ }^{14}$ Zum einen darf es zu keinen Rückschritten im Demokratisierungsprozess kommen, zum anderen ist das Demokratieprinzip ,positiv auf die Erzielung von Fortschritten ausgelegt" ${ }^{15}$ womit auch ein demokratisches Optimierungsgebot besteht.

\section{b) Das europäische Demokratiekonzept und seine Defizite}

Das Verständnis eines europäischen Demokratiekonzepts trägt unterschiedliche Facetten in sich. Es wird dabei Demokratie unabhängig vom nationalstaatlichen Volksbegriff auf europäischer Ebene im Zusammenwirken mit den Mitgliedstaaten konstruiert.

Der Weg zur europäischen Demokratie führt über ein Konzept, das dualistisch die Gesamtheit der Unionsbürgerinnen und -bürger und die mitgliedstaatlich verfassten Völker in die demokratische Legitimation der EU einbezieht. Diese Berücksichtigung der Unionsbürgerinnen und -bürger zeigt bereits einen für direkte Demokratie relevanten Aspekt auf. ${ }^{16}$

Ein darauf aufbauender institutioneller Zugang zu einem europäischen Demokratiekonzept beruht auf zwei einander ergänzenden Legitimationssäulen: dem EP

11 Vgl. dazu näher I. Pernice, in: H. Dreier (Hrsg.), Grundgesetz-Kommentar, Bd. II, 1. Aufl. 1998, Art. 23 Rn. 22, der dabei vor allem auf die schrittweise Konstituierung und demokratische Konsolidierung der europäischen Verträge hinweist; I. Pernice, Europäisches und nationales Verfassungsrecht, in: VVDStRL 60 (2001), S. 148 (165 ff.); zuvor schon J. Frowein, Die Verfassung der Europäischen Union aus der Sicht der Mitgliedstaaten, EuR 1995, 315 (322).

$12 \mathrm{Zu}$ nennen sind hier vor allem die Änderungen durch die Verträge von Maastricht (1992), Amsterdam (1997) und Nizza (2001), die das Mitentscheidungsverfahren bereits als das reguläre Rechtsetzungsverfahren erscheinen lassen. Siehe dazu etwa Klein, Parteienrecht (Fn. 5), S. 541; W. Meng, Institutionen und Verfahren in der „Ersten Säule“ der Europäischen Union nach dem Vertrag von Amsterdam, in: W. Hummer (Hrsg.), Die Europäische Union nach dem Vertrag von Amsterdam, 1998, S. 161 (170 ff.); W. Hummer/W. Obwexer, Der Vertrag von Nizza, 2001, S. 32 ff.; H. Neisser/B. Verschraegen, Die Europäische Union, 2001, Rz. $11.106 \mathrm{ff}$.

13 Vgl. dazu E. Eriksen, Deliberation and the problem of democratic legitimacy in the EU, ARENA WP 2006/8, $20 \mathrm{ff}$.

14 N. Petersen, Demokratie als teleologisches Prinzip. Zur Legitimität von Staatsgewalt im Völkerrecht, 2008, S. $91 \mathrm{ff} ., 138 \mathrm{ff}$.

15 Ebd., S. 139.

16 A. v. Bogdandy, Grundprinzipien, in: ders./Bast, Verfassungsrecht (Fn. 3), S. 13 (63 ff.). 
und den nationalen Parlamenten, vermittelt über den Rat. ${ }^{17}$ Die politische Willensbildung auf Ebene der europäischen Demokratie ist daher neben dem EP auch durch die Regierungen und die nationalen Parlamente vermittelt. ${ }^{18}$ Auf beiden Strängen wird damit - in nationalstaatlich gefärbter Terminologie - ein repräsentativ-demokratisches Modell realisiert. Allerdings ist das EP nicht die zentrale demokratische Drehscheibe der Union, die EU damit nur semi-parlamentarisch. ${ }^{19}$ Mit den institutionellen Defiziten der repräsentativen Demokratie sind aber auch die politischen Grenzen der weiteren Fortbildung der Unionsbürgerschaft erreicht. ${ }^{20}$

Diese Form der Ausgestaltung europäischer Demokratie weist nun angesichts der Besonderheiten europäischer Demokratie, nämlich dem supranationalen Charakter des dabei geschaffenen Rechts, die bekannten und eingehend diskutierten Defizite auf: $:^{21}$

Zum einen ist die Rückkoppelung an die einzelnen „Völker der in der Gemein-

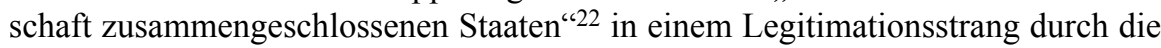
Abhängigkeit der Verantwortlichkeit der Exekutivorgane gegenüber den nationalen Parlamenten sehr stark mediatisiert. Die Ausgestaltung der Repräsentation durch nationale Regierungen im Rat vermittelt in der Praxis das Bild einer primär nationalen Interessenwahrung. ${ }^{23}$ Durch die starke Rolle der Europäischen Kommission als Initiativorgan ${ }^{24}$ und das politische Gewicht von Verwaltungsorganen im Rat zeigt sich ein hohes Maß an „Exekutivlastigkeit der europäischen Rechtsetzung“ ${ }^{25}$

Eine demokratische Repräsentation ist demgegenüber am deutlichsten durch das EP verwirklicht, da die Mandatare des EP durch direkte Wahlen seitens der Unionsbürger legitimiert sind. ${ }^{26}$ Das Wahlrecht für das EP genügt jedoch nicht „den Standards egalitärer Gleichheit“, womit institutionell „,keine egalitär demokratisch legitimierten Mechanismen“ bestehen. ${ }^{27}$ Auch wenn die Vertreter im EP nicht als

17 Siehe dazu C. Calliess, Optionen zur Demokratisierung der Europäischen Union, in: Bauer/ Huber/Sommermann, Demokratie (Fn. 4), S. 281 (282); Nettesheim, Demokratisierung (Fn. 4), S. 178; J. Gerkrath, Die Bedingungen der Demokratie in der Europäischen Union, EuGRZ 2006, 371 (376 f.).

18 Die parlamentarische Demokratie wird als Konzept für die Europäische Union zuweilen sogar in Frage gestellt: siehe dazu m.w.N etwa M. Jachtenfuchs, Democracy and Governance in the European Union, EIoP 1 (1997) No. 2, 6 ff.

19 P. Dann, Die politischen Organe, in: v. Bogdandy/Bast, Verfassungsrecht (Fn. 3), S. 335 (384 f.).

20 S. Kadelbach, Unionsbürgerschaft, in: v. Bogdandy/Bast, Verfassungsrecht (Fn. 3), S. 611 (654).

21 Dazu zusammenfassend U. Haltern, Europarecht, 2. Aufl. 2007, S. 133 ff.

22 Art. 189 Abs. 1 EGV (nunmehr Art. 14 Abs. 2 EUV).

23 Dazu etwa J. Leinen, Europäische Parteien: Aufbruch in eine neue demokratische EU, integration 3/2006, 229 (230).

24 Vgl. v.a. Art. 251 EGV (nunmehr Art. 294 AEUV).

25 Kritisch zum diesbezüglichen Stand und zur Entwicklung der Europäischen Union siehe etwa P. Mair, Popular Democracy and the European Union Polity, European Governance Papers 2005/03.

26 Art. 190 EGV (nunmehr Art. 14 Abs. 2 und 3 EUV).

27 C. Möllers, Verfassungsgebende Gewalt - Verfassung - Konstitutionalisierung, in: v. Bogdandy/Bast, Verfassungsrecht (Fn. 3), S. 227 (253). 
nationale Vertreter verstanden werden, sondern als Interessenwahrer im Verbund transnationaler politischer Organisationen, ${ }^{28}$ so entspricht zum gegenwärtigen Zeitpunkt die Realität nicht dem Idealtypus eines demokratischen Repräsentativorgans. Diese besteht vielmehr im Einfluss von Partialinteressen vertretenden institutionellen Entscheidungsträgern und wirtschaftlichen Interessengruppen, ${ }^{29}$ die das System demokratischer Rückkoppelung an die europäischen Völker geradezu Lügen strafen.

\section{Ansatzpunkte direkter Demokratie im Kontext europäischer Rechtsetzung und Vollziehung}

\section{a) Das Demokratiedefizit als Ausgangspunkt?}

Selbst eine Aufwertung der Rolle des EP, wie sie etwa mit dem unlängst in Kraft getretenen Vertrag von Lissabon ${ }^{30}$ einhergeht, ${ }^{31}$ aber auch die Stärkung der Rolle der nationalen Parlamente ${ }^{32}$ ändert wenig am Befund, dass der Mangel europäischer Demokratie vor allem an der Ferne zwischen den Rechtsetzern und den Normadressaten - dem „Kommunikationsdefizit“ 33 zwischen der Union und den Bürgern - und den mediatisierten Rückkoppelungsmechanismen liegt, also im Effekt an der Zentralisierung des europäischen Demokratiekonzepts.

Man kommt daher nicht umhin, das Maß demokratischer Legitimation europäischer Demokratie in einer Gesamtbetrachtung zu analysieren. Auch aus der Einsicht, dass es kein einheitliches europäisches Volk und damit keine Homogenität europäischer Demokratie gibt und auch nicht geben muss, ${ }^{34}$ ist ein Modell gegliederter Legitimationsebenen als Ansatzpunkt für eine Demokratisierung in den Blick zu nehmen. Dem dient die Sichtweise eines „Mehrebenensystems“, in dem die eu-

28 Zur damit angesprochenen Rolle europäischer politischer Parteien vgl. H. Eberhard/K. Lachmayer, Europäische politische Parteien und deren Finanzierung als Aspekte des europäischen Demokratisierungsprozesses, in: G. Manssen (Hrsg.), Die Finanzierung von politischen Parteien in Europa - Bestandsaufnahme und europäische Perspektive, 2008, S. 215 (219 ff.).

29 I.d.S. etwa $D$. Tsatsos/G. Deinzer, Keine europäische Integration ohne Europäische Politische Parteien, in: dies. (Hrsg.), Europäische Politische Parteien, 1998, S. 13 (13 f.).

30 AB1. 2008/C 115/01 v. 9.5.2008.

31 Der Vertrag von Lissabon macht das Mitentscheidungsverfahren zum „ordentlichen Gesetzgebungsverfahren“ und sieht es damit als Regelverfahren vor (siehe dazu Art. 294 AEUV [Vertrag über die Arbeitsweise der Europäischen Union]).

32 V.a. in der Rolle als „Kontrolleure“ des Subsidiaritätsprinzips. Vgl. dazu die neugefassten Protokolle über die Rolle der nationalen Parlamente einerseits und über die Anwendung der Grundsätze der Subsidiarität und der Verhältnismäßigkeit andererseits. Vgl. zu potenziellen Entwicklungsperspektiven in dieser Hinsicht J. Schwarze, Europäische Verfassungsperspektiven nach Nizza, NJW 2002, 993 (997).

33 V. Neßler, Deutsche und europäische Parteien, EuGRZ 1998, 191 (196).

34 Dazu etwa R. Bieber, Steigerungsform der europäischen Union: Eine Europäische Verfassung, in: FS 180 Jahre Carl Heymanns Verlag, 1995, S. 291 (300 ff.); F. Hanschmann, Der Begriff der Homogenität in der Verfassungslehre und Europarechtswissenschaft, 2008, S. 297 ff.; S. Hammer, Wege zur Europäischen Verfassung, juridikum 2000, 48 (53 f.). 
ropäische und die nationale Ebene einander wechselseitig beeinflussen. ${ }^{35}$ Dieser Kommunikationsprozess läuft auch über die sich im Rahmen der europäischen Integration öffnenden Verfassungsordnungen. Demokratie entfaltet sich daher im europäischen „Verfassungsverbund“"36, der aus der europäischen Verfassung und den nationalen Verfassungen der Mitgliedstaaten besteht. ${ }^{37}$ In diesem Verbund besteht ein „System wechselseitiger Verfassungsbefruchtung und -stabilisierung““.38 Das nationale Verfassungsrecht stellt in diesem System das „,wichtigste Rezeptionsreservoir für das unionale Verfassungsrecht" ${ }^{\text {39 }}$ dar.

Ist das Konzept europäischer Demokratie mit seinem starken repräsentativ-demokratischen Element daher von den nationalen Systemen zu gewissen Teilen abgeleitet und steht mit diesen in einem Austausch, so ist ein weiterer - notwendiger - Entwicklungsschritt in der Konstitutionalisierung Europas, dass auch direkt-demokratische Elemente in die europäische Verfassung Eingang finden sollten. Die Verstärkung der direkt-demokratischen Elemente - als „Absage an einen demokratischen Zentralismus" 40 - könnte einen Beitrag zum Abbau des demokratischen Defizits leisten und damit gleichzeitig eine positive Beeinflussung der Entwicklung des europäischen Parlamentarismus mit sich bringen. Dies soll anhand einer Ebenenbetrachtung im Folgenden beleuchtet werden.

35 Dazu H. Eberhard u.a., Governance - zur theoretischen und praktischen Verortung des Konzepts in Österreich, JRP 2006, 35 (45 f., 54); H. Eberhard/K. Lachmayer, Internationales Verfassungsrecht als internationale Verfassungswissenschaft? Die Betrachtung des GovernanceKonzepts aus der Perspektive des Internationalen Verfassungsrechts, ICL-Working Paper 01/06 (www.icl-workshop.com, 31.8.2009), m.w.N.

36 Zum Begriff des „europäischen Verfassungsverbunds“ siehe Pernice, Verfassungsrecht (Fn. 11), S. 163 ff.

37 Vgl. zu dieser Dimension des Verfassungsverbunds C. Calliess, Das Demokratieprinzip im europäischen Staaten- und Verfassungsverbund, in: FS Ress, 2005, S. 399 (401 ff.); ders., Die Verfassung der EU im Kontext der Erweiterung, in: ders./H. Isak (Hrsg.), Der Konventsentwurf für eine EU-Verfassung im Kontext der Erweiterung, 2004, S. 9 (23 ff.); T. Öhlinger, Gemeinschaftsverfassung und Staatsverfassung, in: E. Busek/W. Hummer (Hrsg.), Etappen auf dem Weg zu einer europäischen Verfassung, 2004, S. 95 (109); C. Grabenwarter, Staatliches Unionsverfassungsrecht, in: v. Bogdandy/Bast, Verfassungsrecht (Fn. 3), S. 121 (173).

38 Vgl. dazu C. Calliess/M. Ruffert, Vom Vertrag zur EU-Verfassung?, EuGRZ 2004, 542 (545): „In einem solchen Verfassungsverbund empfängt die europäische Ebene nicht nur Impulse aus dem mitgliedstaatlichen Verfassungsrecht ..., sondern sendet ebensolche auch dorthin zurück."

39 So P.M. Huber, Europäisches und nationales Verfassungsrecht, VVDStRL 60 (2001), S. 194 (225).

40 P. Bußjäger, Plebiszitäre Demokratie im Mehrebenensystem? - zur Theorie direkter Demokratie in föderalen und konföderalen Systemen, in: FS Pernthaler, 2005, S. 85 (85 f.). 


\section{b) Setzung von Primärrecht}

„Und sind die Iren, dieses kleine Volk von Widerständlern, nicht die einzigen im weiten Europa, die überhaupt nach ihrer Meinung gefragt werden? Sie wollen nicht wie Stimmvieh behandelt werden, das zur Urne getrieben wird."41

Jürgen Habermas wurde aufgrund der aktuellen negativen Volksabstimmungen zu einem Protagonisten der Forderung nach verstärkter (direkt-)demokratischer Konzeption des Europarechts. Im Falle der Volksabstimmungen von Frankreich, der Niederlande und Irlands zeigten sich aber nicht nur die Ignoranz gegenüber direktdemokratischen Entscheidungen, sondern auch deren Konsequenzen. Die Volksabstimmungen in Frankreich und den Niederlanden haben den Verfassungsvertrag zu Fall gebracht und damit den „Verfassungsnominalismus“ der EU beendet. ${ }^{42}$ Die Ablehnung des Vertrags von Lissabon führte zu Zusagen an Irland. Auch wenn man diese Folgen der negativen Volksabstimmungen nicht überbewerten sollte, so sollen diese auch nicht übersehen werden.

Ein Ausbau direkter Demokratie auf Ebene des Primärrechts kann bei jenen Vorgaben ansetzen, welche die Mitgliedstaaten bei der Ratifikation der völkerrechtlichen Charakter besitzenden Vertragsänderungen zu beachten haben. Zumal im Fall des (als solchen gescheiterten) Verfassungsvertrags wurde die Abhaltung eines europaweiten - und idealtypisch gleichzeitigen - Referendums als Perspektive zur Erhöhung der demokratischen Legitimation und Akzeptanz europäischer Verfassunggebung diskutiert. Das bislang geltende Primärrecht ${ }^{43}$ sah vor, dass ausschließlich die Regierung jedes Mitgliedstaats oder die Kommission dem Rat Entwürfe zur Änderung der Verträge, auf denen die Union beruht, vorlegen können. Eine derartige Initiative europäischer Bürger war in diesem Zusammenhang nicht vorgesehen; eine solche fehlt auch im Vertrag von Lissabon. Er sieht jedoch im neuen „ordentlichen“, aber auch im „vereinfachten“ Änderungsverfahren die Erweiterung der antragsberechtigten Institutionen um das EP vor. ${ }^{44}$ Auch die ausschließlich auf die Schaffung von Sekundärrecht abzielende europäische Initiative nach Art. 11 Abs. 4 EUV ${ }^{45}$ i.d.F. des Vertrags von Lissabon läuft für die seitens der Kommission mögliche Erstattung von Vorschlägen für zukünftige Vertragsänderungen leer.

41 J. Habermas, Ein Lob den Iren, Süddeutsche Zeitung v. 16.6.2008 (www.sueddeutsche.de).

42 Möllers, Verfassungsgebende Gewalt (Fn. 27), S. 276.

43 Art. 48 Abs. 1 EUV.

44 Art. 48 Abs. 2 EUV i.d.F. des Vertrags von Lissabon: „Die Regierung jedes Mitgliedstaats, das Europäische Parlament oder die Kommission können dem Rat Entwürfe zur Änderung der Verträge vorlegen. Diese Entwürfe können unter anderem eine Ausdehnung oder Verringerung der der Union in den Verträgen übertragenen Zuständigkeiten zum Ziel haben. Diese Entwürfe werden vom Rat dem Europäischen Rat übermittelt und den nationalen Parlamenten zur Kenntnis gebracht."

Art. 48 Abs 6. EUV i.d.F. des Vertrags von Lissabon: „Die Regierung jedes Mitgliedstaats, das Europäische Parlament oder die Kommission kann dem Europäischen Rat Entwürfe zur Änderung aller oder eines Teils der Bestimmungen des Dritten Teils des Vertrags über die Arbeitsweise der Europäischen Union über die internen Politikbereiche der Union vorlegen. “

45 Dazu sogleich $3 \mathrm{c}$ ). 
Die Einbindung der europäischen Völker über den in den meisten Mitgliedstaaten unterschiedlich ausgestalteten, zumeist jedoch repräsentativ-demokratisch ausgestalteten Ratifikationsprozess überlässt das frühere Primärrecht ${ }^{46}$ wie auch das (nach Inkrafttreten des Vertrags von Lissabon) geltende Primärrecht ${ }^{47}$ der Verfassungsautonomie der Mitgliedstaaten. Ein Referendum über die Änderung von Primärrecht könnte in Anbetracht des Verfassungscharakters dieser Rechtsschicht dem Gedanken eines europäischen pouvoir constituant Rechnung tragen..$^{48}$ Das geltende Primärrecht ist aber erkennbar vom Gedanken getragen, die Unterschiedlichkeit der bestehenden nationalen Ratifikationsmodelle zu respektieren. Ein Ausbau direktdemokratischer Elemente ist auf dieser Ebene daher mittelfristig nicht zu erwarten.

\section{c) Setzung von Sekundärrecht}

Der entscheidende Kern des europäischen Demokratiedefizits wird aber ohnedies auf der Ebene der Schaffung von Sekundärrecht verortet. Das dominierende Organ in der Initialphase europäischer Rechtsetzung auf sekundärrechtlicher Ebene ist die Kommission als „Motor, Wächter und ehrlicher Makler“ (Hallstein).

Direkt-demokratische Elemente können daher folgerichtig an diesem Initiativrecht und institutionell somit an der Kommission anknüpfen. Hier realisiert der Vertrag von Lissabon in Gestalt zweier aufeinander angepasster Bestimmungen einen bemerkenswerten Ausgleich des demokratischen Systems der EU.

Art. 10 EUV in der Fassung des Vertrags von Lissabon proklamiert zum einen über den bisherigen Status des Art. 6 Abs. 1 EUV (Nennung der „Demokratie“ als Grundsatz der $\mathrm{EU}^{49}$ ) hinaus -, dass die Arbeitsweise der Union auf der repräsentativen Demokratie beruht (Abs. 1) und die Bürgerinnen und Bürger auf Unionsebene unmittelbar im Europäischen Parlament vertreten sind (Abs. 2 UAbs. 1). Betont werden als Elemente dieser repräsentativen Demokratie auch die Verantwortlichkeit der Ratsmitglieder gegenüber ihren nationalen Parlamenten (Abs. 2 UAbs. 2) sowie die Rolle der politischen Parteien auf europäischer Ebene für die Herausbildung eines europäischen politischen Bewusstseins und im Hinblick auf den Ausdruck des Willens der Bürgerinnen und Bürger der Union ${ }^{50}$ (Abs. 4).

46 Art. 48 Abs. 3 EUV: „Die Änderungen treten in Kraft, nachdem sie von allen Mitgliedstaaten gemäß ihren verfassungsrechtlichen Vorschriften ratifiziert worden sind.“

47 Dieses sieht im neuformulierten Art. 48 Abs. 4 UAbs. 2 EUV im ordentlichen Änderungsverfahren nahezu wortident zur geltenden Rechtslage vor: ,Die Änderungen treten in Kraft, nachdem sie von allen Mitgliedstaaten nach Maßgabe ihrer verfassungsrechtlichen Vorschriften ratifiziert worden sind.“ Ähnlich Art. 48 Abs. 6 UAbs. 2 EUV für das vereinfachte Änderungsverfahren.

48 Dabei ist die Lehre von der verfassunggebenden Gewalt als ,ein normativ begründeter kritischer Stachel für die Entwicklung einer demokratischen europäischen Integration“ nützlich. Siehe Möllers, Verfassungsgebende Gewalt (Fn. 27), S. 250 ff., 255.

49 Zum Gehalt dieser Bestimmung vgl. etwa W. Hummer, Legitimations- und Demokratiedefizit in der Europäischen Union? Rolle und Funktion des Europäischen Parlaments im ,,institutionellen Gleichgewicht“", in: Busek/Hummer, Etappen (Fn. 37), S. 471 (484 ff.).

50 Vgl. schon bisher Art. 191 EGV. 
Außer diesem Grundsatz verankert Art. 11 EUV in der Fassung des Vertrags von Lissabon Elemente partizipativer Demokratie. ${ }^{51}$ Neben den programmatischen Bestimmungen über die Möglichkeit der Bürgerinnen und Bürger wie der repräsentativen Verbände, in geeigneter Weise ihre Ansichten in allen Bereichen des Handelns der Union öffentlich bekannt zu geben und auszutauschen (Abs. 1), und der Promesse eines „offenen, transparenten und regelmäßigen Dialogs [der Organe der EU] mit den repräsentativen Verbänden und der Zivilgesellschaft" (Abs. 2) stellt sich die europäische Bürgerinitiative (Abs. 4) als Kern des mit dem Vertrag von Lissabon geänderten Demokratiekonzepts dar. ${ }^{52}$ Diese schon auf den europäischen Verfassungskonvent ${ }^{53}$ zurückgehende Bestimmung sieht vor, dass Unionsbürgerinnen und -bürger, deren Anzahl mindestens eine Million betragen und bei denen es sich um Staatsangehörige einer erheblichen Anzahl von Mitgliedstaaten handeln muss, die Initiative ergreifen und die Kommission auffordern können, im Rahmen ihrer Befugnisse geeignete Vorschläge zu Themen zu unterbreiten, zu denen es nach Ansicht jener Bürgerinnen und Bürger eines Rechtsakts der Union bedarf, um die Verträge umzusetzen.

Die Verfahren und Bedingungen, die für eine solche Bürgerinitiative gelten, werden nach Art. 24 Abs. 1 Vertrag über die Arbeitsweise der EU (AEUV) ${ }^{54}$ festgelegt. Dieser wiederum sieht vor, dass die Bestimmungen über die Verfahren und Bedingungen, die für eine Bürgerinitiative i.S.d. Art. 11 EUV gelten, einschließlich der Mindestzahl der Mitgliedstaaten, aus denen die Bürgerinnen und Bürger, die diese Initiative ergreifen, kommen müssen, vom Europäischen Parlament und vom Rat gemäß dem ordentlichen Gesetzgebungsverfahren durch Verordnungen festgelegt werden.

Klargestellt ist damit zum einen, dass mit einem derartigen Bürgerbegehren keine Rechtsakte gesetzt werden können, durch die Kompetenzen der EU erweitert oder verringert würden, weil dies einem primärrechtlichen Akt vorbehalten ist und es sich insoweit nicht um die „Umsetzung der Verträge“ handeln würde. Dies steht zwar in einem gewissen Spannungsverhältnis zur Anordnung, dass das Begehren auf Vorschläge gerichtet sein kann, die „,im Rahmen der Befugnisse“ der Kommission gelegen sind, steht aber in einem systematischen Kontext zum Umstand, dass Vertragsänderungen nach wie vor (und auch weiterhin) einem völkerrechtlichen Verfahren vorbehalten sind bzw. bleiben. ${ }^{55} \mathrm{Im}$ Schrifttum ${ }^{56}$ werden zwar Zweifel gegenüber der Praktikabilität der damit erstmals primärrechtlich vorgesehenen Möglichkeit eines europäischen Bürgerbegehrens geäußert, es steht aber fest, dass damit

51 So lautete in der Fassung des Verfassungsvertrages auch der Titel zum damaligen Art I-47 VVE (ABl. 2004/C 310/01 v. 16.12.2004).

52 Vgl. dazu etwa V. Hellmann, Der Vertrag von Lissabon, 2009, S. 20.

53 Dazu J. Schwarze, Ein pragmatischer Verfassungsentwurf - Analyse und Bewertung des vom Europäischen Verfassungskonvent vorgelegten Entwurfs eines Vertrags über eine Verfassung für Europa, EuR 2003, 535 (556).

54 Die Neubezeichnung für den geänderten Vertrag über die Gründung der Europäischen Gemeinschaft (EGV).

55 Siehe dazu bereits unter $3 \mathrm{~b}$ ).

56 Siehe etwa Schwarze, Verfassungsentwurf (Fn. 53), S. 557. 
auf der symbolträchtigen Ebene des europäischen Verfassungsrechts die Zuordnung des Demokratiemodells zwischen dem Repräsentativsystem und dem bisher nicht vorhandenen direkt-demokratischen System völlig neu vorgenommen wird. Die Effektuierung neuer Instrumentarien hängt auch und vor allem von den Details ab, die zum gegenwärtigen Zeitpunkt noch nicht feststehen.

Es wurde oftmals auf die Funktionsbedingungen europäischer Demokratie hingewiesen: Deren wichtigste ist das Bestehen einer ,europäischen Öffentlichkeit“, die als essenzielles Forum für einen Meinungspluralismus und öffentlichen Diskurs fungiert. ${ }^{57}$ In einer entidealisierten Bestandsaufnahme ist europäische Öffentlichkeit nicht als Einheit, sondern ,als vernetztes System vielfältiger und dezentraler Teilöffentlichkeiten zu denken". ${ }^{58}$ Diese europäischen Teilöffentlichkeiten erfordern auch ein Netz europäischer Medien. ${ }^{59}$ Eben dieser Elemente von europäischer Öffentlichkeit wird auch ein effektiver Einsatz des Bürgerbegehrens bedürfen. Das allgemeine Postulat findet sich damit im speziellen Kontext wieder. In der - durch diese Rahmenbedingungen ermöglichten - Aktivierung eines zivilgesellschaftlichen Elements liegt jedoch ein dynamisches Instrument, das es ermöglichen würde, die Distanz europäischer Rechtsetzung zu ihren Adressaten zu verringern.

\section{d) Ebene der Vollziehung des Unionsrechts}

Wie schon in einem nationalstaatlichen Kontext stellt sich auch im Hinblick auf das Unionsrecht die Frage nach dem Einbau direkt-demokratischer Elemente nicht nur auf Ebene der Rechtsetzung, sondern auch auf jener der Vollziehung. In diesem Zusammenhang ist vorweg zu bemerken, dass für diese Ebene das Trennungsprinzip gilt, welches im Hinblick auf die Vollziehung des Unionsrechts von einem ,indirekten, isoliert-einzelstaatlichen Vollzugsmodell" ${ }^{“ 60}$ ausgeht. In diesem Zusammenhang bestehen zwar unterschiedliche Vorgaben, etwa hinsichtlich der Gleichwertigkeit des Rechtsschutzes in der Vollziehung nationaler und unionsrechtlicher Re-

57 Siehe dazu M. Rau, Overcoming the Democratic Deficit in the European Union - the Neglected Role of Political Parties, in: A. Bodnar u.a. (Hrsg.), The Emerging Constitutional Law of the European Union, 2003, S. 133 (145 f.). Zu den Entwicklungsperspektiven der ,europäischen Öffentlichkeit" siehe Gerkrath, Bedingungen (Fn. 17), S. 374; Kadelbach, Bedingungen (Fn. 8), S. 387.

58 Hanschmann, Homogenität (Fn. 34), S. 236.

59 Vgl. etwa Öhlinger, Europa (Fn. 9), S. 42, zu den Funktionsbedingungen eines europäischen Parlamentarismus: „Ein Parlament funktioniert nicht in einem gesellschaftlichen Vakuum, sondern setzt gesellschaftliche Strukturen voraus: europäische Parteien, europäische Interessenverbände, vor allem aber ein europaweites Kommunikationssystem, dh Medien, die auf einem europäischen Markt angeboten und auch in Anspruch genommen werden und so einen nationenübergreifenden Kommunikationszusammenhang herstellen.“

60 Vgl. dazu E. Schmidt-Aßmann, Das allgemeine Verwaltungsrecht als Ordnungsidee, 2. Aufl. 2004, S. $381 \mathrm{f}$. 
gelung (Äquivalenzprinzip ${ }^{61}$ ), wie sie v.a. in der Rechtsprechung des EuGH entwickelt wurden. Grundsätzlich besteht auf dieser Ebene eine institutionelle und verfahrensrechtliche Autonomie der Mitgliedstaaten, in bestimmten Materien zeigen sich aber auf Ebene dieser nationalen Regelungen deutliche Einflüsse, etwa in den Bürgerbeteiligungsverfahren, wie sie in den letzten 20 Jahren verschiedentlich im Anlagenrecht umgesetzt wurden. ${ }^{62}$ An prominenter Stelle stehen hier die Vorgaben im Bereich der Umweltverträglichkeitsprüfung von Projekten, bei denen mit erheblichen Einflüssen auf die Umwelt zu rechnen ist. ${ }^{63}$

Im Hinblick auf die unmittelbar von EU-Organen vorgenommene, quantitativ allerdings noch untergeordnete Vollziehung von Unionsrecht wurden in den letzten Jahren sehr eingehend die Grenzen der Übertragung von Vollziehungsaufgaben auf Agenturen und andere nicht unmittelbar dem Rat bzw. der Kommission unterstellte Organe diskutiert. ${ }^{64}$ Dabei standen stets Fragen der demokratischen Legitimation und Kontrolle im Mittelpunkt, ${ }^{65}$ nicht jedoch die durch solche Einrichtungen angesichts ihres Aufgabenkreises ${ }^{66}$ durchaus denkbaren Varianten einer Beteiligung von betroffenen Bürgerinnen und Bürgern. Auf der Ebene der Vollziehung von Unionsrecht ergeben sich damit zum gegenwärtigen Zeitpunkt punktuelle, dort jedoch durchaus essenzielle Vorgaben für direkt-demokratische Instrumente auf nationaler Ebene, vor allem in der Umsetzung verfahrensrechtlicher Regelungen.

61 Nach diesem Grundsatz ist beim Vollzug von Unionsrecht durch die Mitgliedstaaten grundsätzlich derselbe Rechtsschutz zu gewähren, wie er im nationalen Recht für nicht unter den Anwendungsbereich des Unionsrechts fallende Angelegenheiten eingeräumt wird: siehe dazu m.w.N etwa T. Öhlinger/M. Potacs, Gemeinschaftsrecht und staatliches Recht, 3. Aufl. 2006, S. $106 \mathrm{f}$.

62 Siehe etwa die Richtlinie 2003/35/EG vom 26.5.2003 über die Beteiligung der Öffentlichkeit bei der Ausarbeitung bestimmter umweltbezogener Pläne und Programme, die u.a. Mindeststandards bei UVP-Verfahren normiert. Vgl. zum Problemkreis dieser Einflüsse im nationalstaatlichen Kontext B. Raschauer, Umweltrecht Allgemeiner Teil, in: N. Raschauer/W. Wessely (Hrsg.), Handbuch Umweltrecht, 2006, S. 13 (26 ff.).

63 Siehe dazu grundlegend die Richtlinie 85/337/EWG vom 27. Juni 1985 über die Umweltverträglichkeitsprüfung bei bestimmten öffentlichen und privaten Projekten. Vgl. zum direktdemokratischen Aspekt dieser Regelungen im nationalstaatlichen Kontext H. Mayer, Plebiszitäre Instrumente in der staatlichen Willensbildung, in: FS 75 Jahre Bundesverfassung, 1995, S. 341 (356).

64 Vgl. etwa W. Hummer, Von der „Agentur“ zum „Interinstitutionellen Amt“, in: FS Öhlinger, 2004, S. 92 (insb. 113 ff.).

65 Dazu etwa A. Orator, Empowering European agencies: Perspectives and limits of European democratic legitimacy, in: H. Eberhard/K. Lachmayer u.a. (Hrsg.), Perspectives and Limits of Democracy, 2008, S. 23 (35 ff.) sowie jüngst C. Görisch, Demokratische Verwaltung durch Unionsagenturen, 2009, S. 185 ff. u. passim.

66 Hummer, Agentur (Fn. 64), S. 111 f.: Es gibt u.a. Informationsagenturen und solche zur Förderung des sozialen Dialogs auf europäischer Ebene. 


\section{Perspektiven post-nationaler Prozesse direkter Demokratie}

Eine Verankerung und ein weiterer Ausbau direkt-demokratischer Instrumente im europäischen Primärrecht und - auf dessen Basis - im Sekundärrecht der EU stellen sich v.a. durch die Verringerung der Distanz von Rechtsetzer und Rechtsadressatenkreis als Mittel zum Abbau des vielerorts monierten „Demokratiedefizits“ der EU und zugleich auch als zentraler inhaltlicher Aspekt der europäischen Verfassungsentwicklung ${ }^{67}$ dar. Das Entwicklungspotenzial für derartige Instrumente ist nach wie vor gegeben, muss sich aber erst zu voller Ausgestaltung hin entwickeln. So hat etwa Joseph Weiler ${ }^{68}$ Vorschläge dahingehend unterbreitet, die Wahlen zum EP mit einer limitierten Anzahl von Referenden zu kombinieren, die durch das EP selbst, durch Mitgliedstaaten oder auch durch ein qualifiziertes Quorum von Bürgerinnen und Bürgern initiiert werden könnten. Ausbaumöglichkeiten finden sich damit sowohl auf der Ebene europäischer Rechtsetzung als auch der Vollziehung.

In historischer Betrachtung zeigt sich, dass sich die Demokratisierung als Kernelement der Konstitutionalisierung der EU vor allem in den traditionellen Bahnen repräsentativer Demokratie entwickeln musste, weil die Verfassungsordnungen der Mitgliedstaaten der EU diese Systementscheidung getroffen haben und direkt-demokratische Elemente auch dort eher als unterstützende Ergänzung fungieren. Direkte Demokratie ist auch und vor allem deswegen auf der Ebene der EU - mehr noch als auf nationaler Ebene - ein Instrument, das beträchtliches Ausbaupotenzial besitzt. Auch hier sind aber mit den im Vertrag von Lissabon enthaltenen Bestimmungen über Instrumente der ,partizipativen Demokratie“ (Art. 11 EUV i.d.F. dieses Vertrages) und vor allem mit der erstmaligen Verankerung einer europäischen Bürgerinitiative die Gewichte in der Relation von repräsentativer und direkter Demokratie in signifikanter Weise neu austariert worden.

Einmal mehr ist zu betonen, dass sich die europäische Demokratie - trotz der Rückkoppelungsprozesse im ,europäischen Verfassungsverbund“ - in einer autonomen Weise entwickelt. Dies bedeutet, dass nationale Regelungen kein direkter Maßstab für die auf europäischer Ebene realisierte demokratische Legitimation sind. Diese Tendenz zum Rekurs auf den nationalen Legitimationsmaßstab besteht aber - wie zuletzt auch das Urteil des deutschen Bundesverfassungsgerichts ${ }^{69}$ zum Vertrag von Lissabon erkennen lie $\beta-$ nach wie vor. ${ }^{70}$

67 S. Griller, Der ,Sui Generis Charakter' der EU und die Konsequenzen für die Verfassungsoptionen, in: W. Hummer (Hrsg.), Paradigmenwechsel im Europarecht zur Jahrtausendwende, 2004, S. $23(37,40)$.

68 J.H.H. Weiler, The Constitution of Europe, 1999, S. $350 \mathrm{f}$.

69 BVerfG 30.6.2009, 2 BvE 2/08, 2 BvE 5/08, 2 BvR 1010/08, 2 BvR 1022/08, 2 BvR 1259/08 und 2 BvR 182/09, Rn. 289 f.: „Das - gemessen an staatlichen Demokratieanforderungen bestehende Defizit der europäischen Hoheitsgewalt kann durch andere Regelungen des Vertrags von Lissabon nicht aufgewogen und insoweit nicht gerechtfertigt werden. [...] Die Europäische Union versucht, die bestehende erhebliche Überföderalisierung auszugleichen, vor allem durch die Stärkung der auf Beteiligung und auf Transparenz zielenden Rechte, sowohl der Bürger wie der Verbände, als auch durch eine Aufwertung der nationalen Parlamente und der Regionen. Der Vertrag von Lissabon stärkt diese auf verfahrensrechtliche Beteiligung ge- 
Die Verankerung des europäischen Bürgerbegehrens durch den Vertrag von Lissabon hat eine nicht zu unterschätzende konstitutionelle Symbolkraft und stellt insoweit ein Förder- und Leitinstrument für die weitere autonome Entwicklung des europäischen Demokratiemodells dar. Hierfür ist allerdings auch eine entsprechende direkt-demokratische Kultur innerhalb der EU zu entwickeln, für die das Diktum von der „Aktivierung einer europäischen Zivilgesellschaft" ${ }^{\text {"71 }}$ keine Worthülse bleibt. Transparenz, Partizipation und Deliberation werden in dieser Hinsicht zu den entscheidenden Perspektiven im europäischen Demokratieprozess. ${ }^{72}$ Post-national ausgestaltete direkte Demokratie kann damit Teil eines demokratischen Diskussionsprozesses vernetzter europäischer Teilöffentlichkeiten werden.

richteten partizipativen Demokratiegehalte. Neben den Elementen ergänzend partizipativer Demokratie, wie dem Gebot, den Unionsbürgern und ,repräsentativen ‘ Verbänden in geeigneter Weise die Möglichkeit zu geben, ihre Ansichten zu kommunizieren, sieht der Vertrag von Lissabon auch Elemente assoziativer und direkter Demokratie vor (Art. 11 EUV-Lissabon). Hierzu zählen der Dialog der Unionsorgane mit ,repräsentativen“ Verbänden und der Zivilgesellschaft sowie die europäische Bürgerinitiative. Sie ermöglicht es, die Kommission unverbindlich aufzufordern, zu politischen Themen geeignete Regelungsvorschläge zu unterbreiten. Eine solche Aufforderung unterliegt einem Quorum von mindestens einer Million Unionsbürgern, die aus einer, erheblichen Anzahl von Mitgliedstaaten' stammen müssen (Art. 11 Abs. 4 EUV-Lissabon). Die Bürgerinitiative ist auf Gegenstände im Rahmen der Zuständigkeit der Kommission begrenzt und bedarf der sekundärrechtlichen Ausgestaltung durch eine Verordnung (Art. 24 Abs. 1 AEUV). Die europäische Bürgerinitiative gilt zugleich als Maßnahme, die in der Erklärung von Laeken angemahnte Bildung einer europäischen Öffentlichkeit zu befördern.“

70 ,The vision of representative democracy that is used in discussions on the legitimacy of European institutions often seems to have more to do with eighteenth-century models of democracy than with the governance of complex post-industrial societies“. R. Dehousse, Beyond representative democracy: constitutionalism in a polycentric polity, in J. Weiler/M. Wind (Hrsg.), European Constitutionalism Beyond the State, 2003, S. 135 (137).

71 Dazu etwa Schwarze, Verfassungsentwurf (Fn. 53), S. 557.

72 Siehe dazu etwa R. Dehousse, Representative democracy (Fn. 70), S. 150 ff.; v. Bogdandy, Grundprinzipien (Fn. 16), S. 66 ff.; v. Bogdandy betont zu Recht, dass Partizipation dem Prinzip politischer Gleichheit dienen muss. 